\title{
Discussion: Limit void ratios and steady-state line of non-plastic soills
}

Luis Alberto Torres-Cruz BSc, PhD

Senior Lecturer, School of Civil and Environmental Engineering, University of the Witwatersrand, Johannesburg, South Africa

(corresponding author: luisalberto.torrescruz)

(Orcid:0000-0001-8482-0070)
Debdeep Sarkar MSC

Doctoral candidate, Ruhr-Universität Bochum, Bochum, Germany (Orcid:0000-0002-2470-2934)

Meisam Goudarzy PhD

Postdoctoral Research Fellow, Ruhr-Universität Bochum, Bochum, Germany

\section{Contribution by D. Sarkar and M. Goudarzy}

In a topic of considerable interest to the research community, Torres-Cruz (2018) made an attempt to reconcile the parameters of the commonly adopted steady-state equation (intercept $\Gamma$ and slope $\lambda$ ) with the limiting void ratios $e_{\max }$ and $e_{\min }$. The author must be lauded for the tedious task of accumulating a huge database of 149 non-plastic soil types for the basis of the research. While the paper has some interesting correlations and inferences, a couple of observations are vague and contradictory to observations made by the contributors and further clarification is requested.

Considering an example, the author argues for the adaptation of empirical equations based on $\lambda$ and $e_{\min }$ as in Figure 3(b) of the original paper (Torres-Cruz, 2018), reasoning that the fines content bias is minimised. However, the data scatter is considerable and an empirical model with $R^{2}=0 \cdot 17$ is not a particularly good representation and raises validity concerns. The correlations do not consider morphological factors such as particle size, shape and roughness, which may explain the considerable scatter in the datasets, therefore rendering a comparison based solely on $\Gamma$ and $\lambda$ unreliable. Had the comparisons been facilitated on materials having similar morphological factors, such an observation would have been more meaningful.

In addition, the author's argument of the comparison between Figures 1 and 4(a) of the original paper is vague. True, the definition of fines is arbitrarily given, but it must be noted that the scatter arises not only due to varying fines content, but also due the particle morphology in general. Consider, for example, when particles are angular in nature, due to high interlocking between individual grains, the limit void ratios are considerably higher than rounded materials, which lack interlocking (Cho et al., 2006; Sarkar et al., 2018). This hypothesis can be extended in the case of non-plastic fines as well. Thus, one could reasonably expect that the values of the slope and intercept for angular materials are considerably higher than those for rounded materials. Such an observation is supported by experimental observations by the contributors and also documented elsewhere (Jerves et al., 2016). So, the observation that the scatter observed in Figure 4 is only due to the broadness of the particle size distribution (PSD) and similar particle shape is questionable.

\section{Authors' reply}

The acknowledgement by Sarkar and Goudarzy regarding the effort that went into compiling the database is appreciated, and it is hoped that the database will continue to grow and be useful to others. The contributors requested clarification regarding two aspects of the paper.

The first is the questionable validity of the $\lambda-e_{\min }$ correlation due to the high underlying scatter (Figure 3(b)). The paper acknowledges that this correlation is indeed weak. Furthermore, Section 5 points out the large uncertainties that result when estimating $\lambda$ from Equation 3 and suggests that both Equations 2 and 3 be used to make qualitative predictions only. The discussers indicate that part of the scatter may arise because morphological particle characteristics such as size and shape have not been taken into account. However, the premise of the paper is that $\Gamma_{100}-e_{\min }$ and $\lambda-e_{\min }$ correlations are plausible because $e_{\min }$ reflects the combined effects of PSD and particle shape (Table 1). That is, the effects of PSD and particle shape are implicitly accounted for through $e_{\min }$. This is consistent with the overlap between angular and rounded soils in $\Gamma_{100}-e_{\min }$ and $\lambda-e_{\min }$ space (Figure 7). It also explains why an approximately unique $\Gamma_{100}-e_{\min }$ correlation is observed in soils with marked differences in PSD (e.g. Figures $8(\mathrm{c})$ and $8(\mathrm{~g})$ ).

Accepting that $e_{\min }$ captures the effect of particle shape and PSD, then what generates the high scatter in the $\lambda-e_{\min }$ space? Two possibilities are suggested in the original paper: the effect of mineralogy and the greater uncertainty on $\lambda$ than on $\Gamma_{100}$. This latter point is further illustrated in Figure 10, which shows the steady-state lines (SSLs) of two mixtures of Hokksund sand and Chengbei silt reported by Yang et al. (2006) (Table 2). These mixtures have fines contents (FC) of $20 \%$ and $94 \%$ and will be referred to as HCM20 and HCM94, respectively. The SSL of HCM20 is better defined than that of HCM94. Accordingly, the relative standard errors (RSEs) of $\Gamma_{100}$ and $\lambda$ are lower for HCM20 than for HCM94. More 


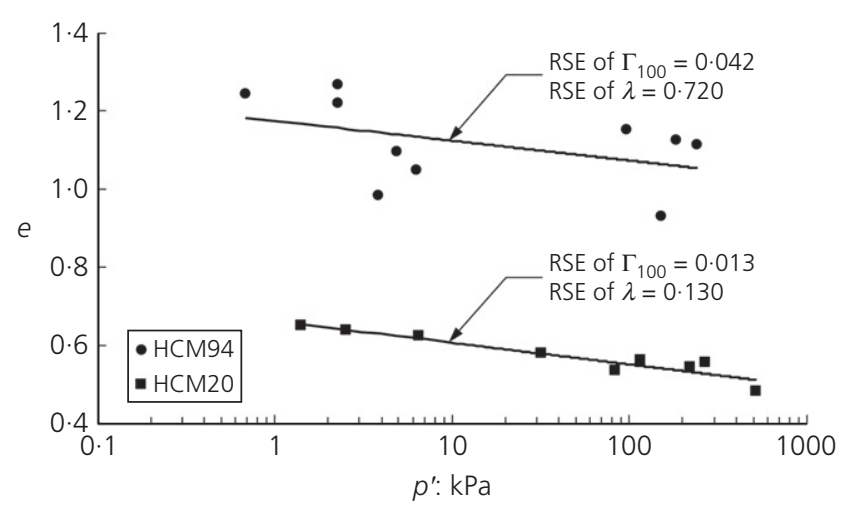

Figure 10. SSLs of HCM2O and HCM94 (data from Yang et al. (2006))

relevant to this discussion, for both soils, the RSE of $\Gamma_{100}$ is significantly lower than the RSE of $\lambda$. That is, there is greater certainty on the estimate of $\Gamma_{100}$ than of $\lambda$. This is generally true for the entire database, not only for the two SSLs shown in Figure 10. The comparison made in the paper between the average RSE of $\lambda(0 \cdot 21)$ and the average RSE of $\Gamma_{n}(0 \cdot 012$ to $0 \cdot 032$, Figure 2) highlighted this point. The high RSE of $\lambda$ may hinder the development of strong correlations between $\lambda$ and soil index properties using this database.

The second aspect to clarify is the comparison between Figures 1 and 4(a). The paper shows that there is no correlation between $\Gamma_{100}$ and FC (Figure 4(a)). This observation disagrees with studies showing a systematic $\Gamma_{n}-\mathrm{FC}$ correlation of the type presented in Figure 1. In the original paper, the comments relating to the comparison of these figures aimed to explain why a correlation is observed in Figure 1 but not in Figure 4(a). The discussers correctly point out that variations in particle morphology contribute to the scatter in Figure 4(a). Likewise, variations in the PSD also contribute to the scatter. For example, if two soils have identical FC, but have differences in particle shape or PSD, or both, the soils will likely have different SSLs. Accordingly, there is no reason to expect a unique correlation between FC and $\Gamma_{100}$. This is in stark contrast to what is observed when laboratory mixes are created from a given sand and a given silt mixed in different proportions. In such cases, the FC is uniquely correlated to the PSD and particle shape distribution. The result is a welldefined $\Gamma_{100}-\mathrm{FC}$ correlation (Figure 1), which may create the impression that the FC plays a key role in defining $\Gamma_{100}$. Comparison of Figures 1 and 4(a) makes it clear that the apparent effect of $\mathrm{FC}$ on $\Gamma_{100}$ disappears when considering the entire database because the FC is no longer uniquely correlated to PSD or particle shape.

The paper relies on $e_{\min }$ to account for the effect of particle shape and PSD. The resulting correlations are expected to be useful when selecting samples that allow the characterisation of SSL variability of a wide variety of non-plastic soil deposits. However, research into the isolated effects of particle shape and PSD continues to be important from a fundamental perspective and for more specific applications.

\section{REFERENCES}

Cho GC, Dodds J and Santamarina JC (2006) Particle shape effects on packing density, stiffness, and strength: natural and crushed sands. Journal of Geotechnical and Geoenvironmental Engineering 132(5): 591-602.

Jerves AX, Kawamoto RY and Andrade JE (2016) Effects of grain morphology on steady state: a computational analysis. Acta Geotechnica 11(3): 493-503, https://doi.org/10.1007/ s11440-015-0422-8.

Sarkar D, Goudarzy M and König D (2018) On the influence of particle characteristics on the index void ratios in granular materials. Particuology (in press).

Torres-Cruz LA (2018) Limit void ratios and steady-state line of non-plastic soils. Proceedings of the Institution of Civil Engineers - Geotechnical Engineering, https://doi.org/ 10.1680/jgeen.18.00011.

Yang SL, Sandven R and Grande L (2006) Steady-state lines of sand-silt mixtures. Canadian Geotechnical Journal 43(11): 1213-1219. 